OPEN ACCESS

Approved by:

Frontiers Editorial Office,

Frontiers Media SA, Switzerland

*Correspondence:

Fengtao Huang

huang_fengtao@126.com

Longfei Wang

wanglf@whu.edu.cn

Bin Zhu

bin_zhu@hust.edu.cn

Specialty section:

This article was submitted to

Phage Biology

a section of the journal

Frontiers in Microbiology

Received: 17 December 2021 Accepted: 20 December 2021

Published: 11 January 2022

Citation:

Huang F, Lu X, Yu C, Sliz P, Wang L and Zhu $B$ (2022) Corrigendum: Molecular Dissection of the Primase

and Polymerase Activities of

Deep-Sea Phage NrS-1

Primase-Polymerase.

Front. Microbiol. 12:838050.

doi: 10.3389/fmicb.2021.838050

\section{Corrigendum: Molecular Dissection of the Primase and Polymerase Activities of Deep-Sea Phage NrS-1 Primase-Polymerase}

\author{
Fengtao Huang ${ }^{1 *}$, Xueling Lu ${ }^{1}$, Chunxiao Yu ${ }^{2}$, Piotr Sliz ${ }^{2}$, Longfei Wang ${ }^{2,3,4 *}$ and Bin Zhu ${ }^{1 *}$ \\ ${ }^{1}$ Key Laboratory of Molecular Biophysics, The Ministry of Education, College of Life Science and Technology and Shenzhen \\ College, Huazhong University of Science and Technology, Wuhan, China, ${ }^{2}$ Department of Biological Chemistry and Molecular \\ Pharmacology, Harvard Medical School, Boston, MA, United States, ${ }^{3}$ Program in Cellular and Molecular Medicine, Boston \\ Children's Hospital, Boston, MA, United States, ${ }^{4}$ School of Pharmaceutical Sciences, Wuhan University, Wuhan, China
}

Keywords: DNA polymerase, primase, DNA replication, de novo synthesis, phage

\section{A Corrigendum on}

Molecular Dissection of the Primase and Polymerase Activities of Deep-Sea Phage NrS-1 Primase-Polymerase

by Huang, F., Lu, X., Yu, C., Sliz, P., Wang, L., and Zhu, B. (2021). Front. Microbiol. 12:766612. doi: $10.3389 /$ fmicb.2021.766612

In the published article, there was an error regarding the affiliations for Longfei Wang. As well as having affiliations 2 and 3 , they should also have affiliation 4 .

The authors apologize for this error and state that this does not change the scientific conclusions of the article in any way. The original article has been updated.

Publisher's Note: All claims expressed in this article are solely those of the authors and do not necessarily represent those of their affiliated organizations, or those of the publisher, the editors and the reviewers. Any product that may be evaluated in this article, or claim that may be made by its manufacturer, is not guaranteed or endorsed by the publisher.

Copyright $(2022$ Huang, Lu, Yu, Sliz, Wang and Zhu. This is an open-access article distributed under the terms of the Creative Commons Attribution License (CC BY). The use, distribution or reproduction in other forums is permitted, provided the original author(s) and the copyright owner(s) are credited and that the original publication in this journal is cited, in accordance with accepted academic practice. No use, distribution or reproduction is permitted which does not comply with these terms. 